\title{
Towards a Linear Combination of Dichotomizers by Margin Maximization
}

\author{
Claudio Marrocco, Mario Molinara, \\ Maria Teresa Ricamato, and Francesco Tortorella \\ DAEIMI - Università degli Studi di Cassino, Cassino, Italy \\ \{c.marrocco,m.molinara,mt.ricamato, tortorella\}@unicas.it
}

\begin{abstract}
When dealing with two-class problems the combination of several dichotomizers is an established technique to improve the classification performance. In this context the margin is considered a central concept since several theoretical results show that improving the margin on the training set is beneficial for the generalization error of a classifier. In particular, this has been analyzed with reference to learning algorithms based on boosting which aim to build strong classifiers through the combination of many weak classifiers. In this paper we try to experimentally verify if the margin maximization can be beneficial also when combining already trained classifiers. We have employed an algorithm for evaluating the weights of a linear convex combination of dichotomizers so as to maximize the margin of the combination on the training set. Several experiments performed on publicly available data sets have shown that a combination based on margin maximization could be particularly effective if compared with other established fusion methods.
\end{abstract}

Keywords: Multiple Classifier Systems, Two-class classification, Margins, Linear Combination.

\section{Introduction}

In order to improve the classification performance a widely used technique to construct effective systems in many real world problems consists in combining more classifiers so as to take advantage of the strengths of the single classifiers and avoid their weaknesses. Combination is therefore considered a profitable solution in applications where some single classifiers are already available and their fusion could represent a convenient way to obtain a more proficient classification systems without building a new classifier from the scratch.

A huge number of possible combination rules has been proposed up to now [10. Among them, the linear combination is certainly the most popular choice for application purposes due to both its simplicity and effectiveness [6] and for this reason it has been thoroughly studied to provide a theoretical model of its behavior with respect to the performance of the combined classifiers and the correlation among their outputs 6[16].

This can be particularly useful in two-class problems that require highly discriminating classifiers. In this context linear combiners are generally built at the 
aim of minimizing the classification error and guarantee a good generalization degree of the recognition/verification system.

In machine learning field the issue of generalization has been particularly analyzed to explain the reduction of the generalization error achieved by Adaboost. To this aim, Schapire et al. [14 introduced the concept of margin which can be considered as a measure of the classification confidence. More precisely, while the sign of the margin indicates the correctness of the classifier, the magnitude estimates the confidence of the classifier in making its prediction on a given sample. Moreover, the margin has a direct connection with the generalization capability of the classifier as proved in several papers [1315] which suggested upper bounds on generalization error expressed in terms of the classifier margin.

In 12 we focused on the concept of margin to evaluate the weights of a convex combination able to maximize the minimum margin of the combination on the training set of a multibiometrics problem. Starting from this point we further analyze the concept of margin to increase the generalization performance of the algorithm. To this aim, in this paper we introduce a soft margin-based combination rule that relaxes the constraint of the optimization problem allowing the system to achieve much better generalization results. Maximizing the smallest soft margin, we do not force outlier values to be assigned to their possible wrong label but we allow for some errors so as to find a trade off between margin and the contribution of each pattern to the decision. Several experiments performed on publicly available data sets have shown that this method is particularly effective if compared with other established linear fusion methods.

The rest of the paper is organized as follows: in sect. 2 the concept of margin and the difference between hard and soft margin are briefly shown while in sect. 3 the method for maximizing the minimum soft margin is described. In sect. 4 the results obtained on some benchmark data sets are presented and discussed. and finally, in sect. 5 some conclusions and an outline of further researches are briefly reported.

\section{Margins and Generalization}

We consider a standard two-class classification problem defined on a training set $S$ containing $N$ samples $\left(\mathbf{x}_{i}, y_{i}\right), i=1, \cdots, N$ where $\mathbf{x}_{i}$ belongs to an instance space $X$ and the labels $y_{i}$ to the set $\{-1,+1\}$. A classifier $f$ can be described as a mapping from $X$ to the interval $[-1,+1]$ such that a sample $\mathbf{x}_{i} \in X$ is assigned to one of the classes according to $\operatorname{sgn}\left(f\left(\mathbf{x}_{i}\right)\right)$. If we assume that $y_{i}$ is the correct label of $\mathbf{x}_{i}$, the sample margin (or hard margin) associated to $\mathbf{x}_{i}$ is given by $y_{i} f\left(\mathbf{x}_{i}\right)$. As a consequence $f$ provides a wrong prediction for $\mathbf{x}_{i}$ if the sample margin is negative and therefore the margin can be interpreted as a measure of the confidence in the prediction of the combined classifier.

The margin theory has been widely used in ensemble learning to analyze the generalization effectiveness of Adaboost. As it is well known, this algorithm generates weak classifiers via dinamically reweighting training samples on the basis of previous classification results; the final classifier is obtained by a convex combination of the constructed weak classifiers. In particular, in [14 it has been 
observed that AdaBoost continues to train even after the training error of the combination is zero that implies an improvement in the generalization error. It has been also proved that this behavior is due to the continue increasing of the margins of the samples in the training set. Some theoretical bounds have been determined on the generalization error of linear classifiers 14,4,9] showing that it improves with the classifier margin (or minimum margin), i.e. with the smallest margin of a sample over the training set $S: \mu(f)=\min _{i} y_{i} f\left(\mathbf{x}_{i}\right)$. The classifier margin has an intuitive meaning [5]: it measures the distance that the classifier can travel in the feature space without changing the way it labels any of the sample points. Therefore the size of the minimum margin (i.e. the margin related to the worst result on the training set) appears to be the most relevant factor for improving generalization.

On this basis, some experiments have been done aimed at improving the generalization performance of Adaboost by using a combination of the generated classifiers explicitly based on minimum margin maximization [7. In 4] Breiman proposed a modified algorithm, called Arc-GV, suitable for this task showing that it asymptotically maximizes the margin. However, the results showed that these modified versions of Adaboost perform generally worse than the original one. A possible reason is that the boosting approach tries to improve their margins focusing on the most difficult samples but with a significant possibility of overfitting when dealing with very difficult samples [13. This problem could be further emphasized by a minimum margin maximization combination which would give higher weights to the classifiers with higher minimum margins, but more prone to overfitting. Therefore, we need to relax the hard margin and allow for a possibility of mistrusting the data. To this aim, it is possible to introduce a non-negative quantity $\xi\left(\mathbf{x}_{i}\right)$ which expresses the possible mistrust on a sample $\mathbf{x}_{i}$ and define the soft margin as a trade off between the hard margin and $\xi\left(\mathbf{x}_{i}\right)$ : $y_{i} f\left(\mathbf{x}_{i}\right)+C \xi\left(\mathbf{x}_{i}\right)$. In this way we can again focus on the minimum margin but considering now the smallest soft margin so expecting to observe less overfitting with respect to the hard margin. As a final remark it is worth noting that the idea of minimizing margins is reminiscent of the idea of support vector machines [18. There, however, one tries to find a linear combination that achieves the best worst separation in the sense of Euclidean and therefore it turns out that SVMs maximize margins using quadratic rather than linear programs.

However, all these studies have only been focused on Boosting algorithms which construct from scratch an ensemble of classifiers as different instances of the same base learning algorithm. As far as we know, the potential effectiveness of such a combination has not yet been examined when the classifiers of the ensemble are built independently and not according to a boosting approach.

\section{Soft Margin Maximization via Linear Programming}

Let us now extend the concept of margin to the combination of $K$ already trained classifiers $f_{j}(\mathbf{x}) \rightarrow[-1,+1]$ with $j=1, \cdots, K$. The hard margin provided by the $j$-th classifier over the $i$-th sample is defined as: 


$$
\mu_{i j}=y_{i} \cdot f_{j}\left(\mathbf{x}_{i}\right) \quad \forall i=1, \ldots, N, \quad \forall j=1, \ldots, K
$$

so that the $f_{j}$ correctly classifies $\mathbf{x}_{i}$ iff $\mu_{i j}>0$. If we now consider the linear convex combination of the $K$ classifiers:

$$
f_{c}(\mathbf{x})=\sum_{j=1}^{K} w_{j} f_{j}(\mathbf{x})
$$

where $w_{j} \geq 0$ and $\sum_{j=1}^{K} w_{j}=1$, the hard margin provided by $f_{c}$ over the $i$-th sample is

$$
\mu_{i}=y_{i} \cdot\left(\sum_{j=1}^{K} w_{j} f_{j}\left(\mathbf{x}_{i}\right)\right)=\sum_{j=1}^{K} w_{j} \mu_{i j} \quad \forall i=1, \ldots, N
$$

while the hard margin of $f_{c}$ is $\mu=\min _{i} \mu_{i}$. However, as said in the previous section, the hard margin could not generalize well on noisy data and therefore we consider the maximization of the soft margin. Since $\mu$ depends on the weights $\mathbf{w}=\left\{w_{1}, w_{2}, \cdots, w_{K}\right\}$, we can choose such weights to make the hard margin as large as possible penalizing it with positive slack variables $\xi_{i}$. In this way we have a max-min problem which can be written as:

$$
\begin{aligned}
& \operatorname{maximize}\left(\min _{i} \sum_{j=1}^{K} w_{j} \mu_{i j}-C \sum_{i=1}^{N} \xi_{i}\right) \\
& \text { subject to } \quad \sum_{j=1}^{K} w_{j}=1 \\
& \begin{array}{ll}
w_{j} \geq 0 & \forall j=1, \ldots, K \\
\xi_{i} \geq 0 & \forall i=1, \ldots, N
\end{array}
\end{aligned}
$$

The penalizing term in the objective function is introduced to avoid large values for the slack variables $\xi$. This allows that some samples have margins larger than $\mu$ according to the value of the trade off constant $C$.

The problem can be recast as a linear problem [17] if we introduce the margin $\mu$ as a new variable:

$$
\begin{array}{cc}
\underset{\text { maximize }}{\operatorname{mubject} \text { to }} & \mu-C \sum_{i=1}^{N} \xi_{i} \\
\\
\qquad \begin{array}{cc}
\sum_{j=1}^{K} w_{j} \mu_{i j} \geq \mu-\xi_{i} & \forall i=1, \ldots, N \\
\sum_{j=1}^{K} w_{j}=1 & \\
w_{j} \geq 0 & \forall j=1, \ldots, K \\
\xi_{i} \geq 0 & \forall i=1, \ldots, N
\end{array}
\end{array}
$$


Let us now collect the margins in a $N \times K$ matrix $\mathbf{M}=\left\{\mu_{i j}\right\}$, the weights in a vector $\mathbf{w}$, the slack variables in a vector $\boldsymbol{\xi}$ and let us define $\mathbf{e}_{t}$ as the column vector consisting of $t$ ones and $\mathbf{z}_{t}$ as the column vector consisting of $t$ zeros. We can then rewrite the problem in vectorial notation:

$$
\begin{array}{cc}
\underset{\text { subject to }}{\operatorname{maximize}} & \mu-C \mathbf{e}_{N}^{T} \boldsymbol{\xi} \\
\mu \mathbf{e}_{N}-\mathbf{M} \mathbf{w}-\boldsymbol{\xi} \leq \mathbf{z}_{N} \\
\mathbf{e}_{K}^{T} \mathbf{w}=1 \\
\mathbf{w} \geq \mathbf{z}_{K} \\
\boldsymbol{\xi} \geq \mathbf{z}_{N}
\end{array}
$$

Finally, considering $\mathbf{I}_{t}$ as the $t$-dimensional identity matrix we can write the problem in block-matrix form:

$$
\begin{aligned}
& \text { maximize }\left[\begin{array}{ll}
\mathbf{z}_{K}^{T}-C \mathbf{e}_{N}^{T} & 1
\end{array}\right]\left[\begin{array}{l}
\mathbf{w} \\
\boldsymbol{\xi} \\
\mu
\end{array}\right] \\
& \text { subject to } \\
& \left.\qquad \begin{array}{ccc}
-\mathbf{M}-\mathbf{I}_{N} & \mathbf{e}_{N} \\
\mathbf{e}_{K}^{T} & \mathbf{z}_{N}^{T} & 0
\end{array}\right]\left[\begin{array}{c}
\mathbf{w} \\
\boldsymbol{\xi} \\
\mu
\end{array}\right] \leq\left[\begin{array}{c}
\mathbf{z}_{N} \\
1
\end{array}\right] \\
& \mathbf{w} \geq \mathbf{z}_{K} \\
& \boldsymbol{\xi} \geq \mathbf{z}_{N}
\end{aligned}
$$

As a final remark, it is worth noting that to solve this problem we could use any one of the numerous linear programming methods available. However, it should be taken into account that the number of constraints could be very large since it equals the number of training samples.

\section{Experiments}

The effectiveness of the proposed fusion rule has been evaluated on 10 twoclass data sets taken from the UCI Repository of Machine Learning Database 2 with different sizes and class skew. The features were previously scaled so as to have zero mean and unit standard deviation. To avoid any bias due to the particular partition of data set, 10 runs of a multiple hold out procedure have been performed for each data set. In each run, the data set has been parted into three sets: a training set to train the classifier, a tuning set to estimate the optimal weights (i.e. to train the combiner) and a test set to evaluate the compared combination methods.

The soft margin fusion rule (hereafter called SM) has been compared to the hard margin fusion rule (HM) and to two single combination rules: the simple 


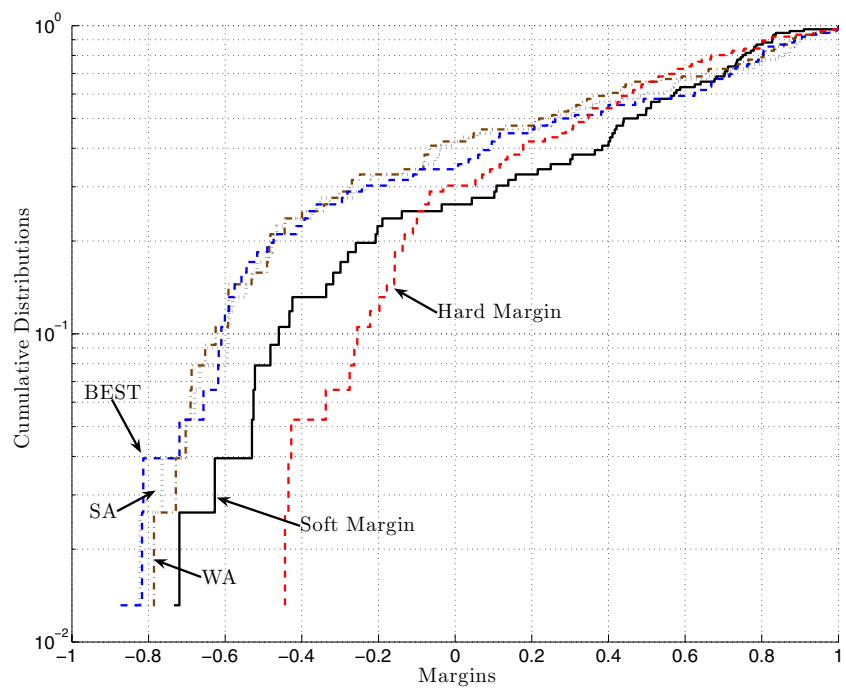

(a)

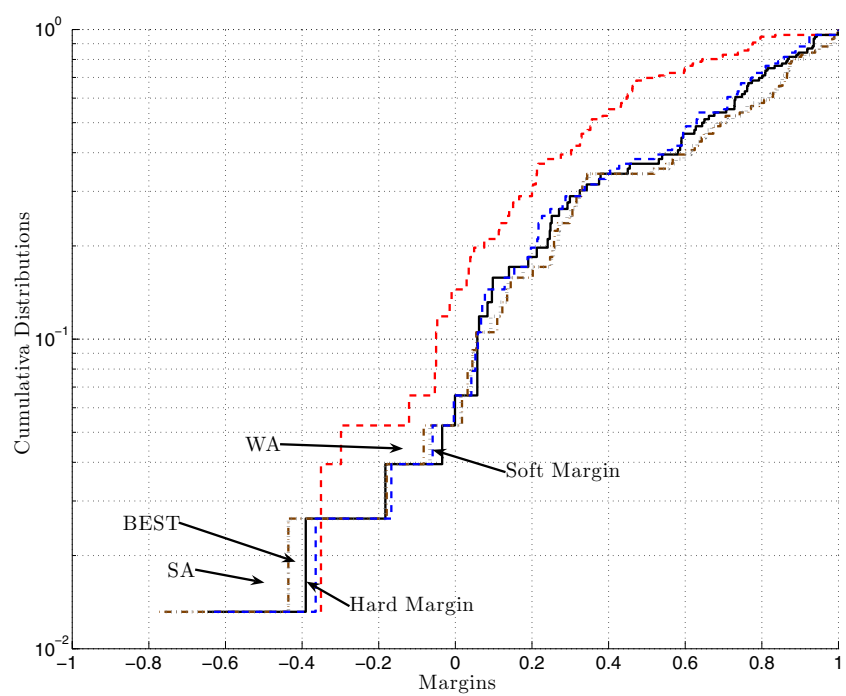

(b)

Fig. 1. Margin distributions graphs for the employed combination rules on the House data set when using WRLC (a) and ADAR (b) classifiers. The scale on y-axis is logarithmic. 
average (SA) and the weighted average (WA) defined as $f(x)=\sum_{j=1}^{K} w_{j} f_{j}(x)$ where $w_{j}=\frac{\text { Error }_{j}}{\sum_{j=1}^{K} \text { Error }_{j}}$, i.e. where the classifiers are weighted according to the provided error rate. For the sake of completeness the results obtained by the best base classifier (BEST in the following tables) employed in each combination have been reported. All the data in input to the combiner have been normalized using the min-max normalization $[8$. Then, to assign a sample to the positive or negative class a threshold to be imposed on the output of the combiner has been evaluated on the tuning set. In particular, using the Receiver Operating Characteristic (ROC) curve we determined the threshold value that minimizes the empirical error rate on the tuning set.

As base classifiers, we choose two different models. The first one is a random linear classifier, called Weak Random Linear Classifier (WRLC) [11] that allows us to easily build several simple classifiers by randomly assigning the coefficients of the linear discriminant function. If the error rate evaluated on the training set is lower than or equal to 0.5 the coefficients are held, otherwise the signs of the coefficients are reversed so as to invert the outputs of the classifier and ensure an error rate lower than 0.5. The second model is an AdaBoost classifier, implemented by means of the Modest AdaBoost algorithm [19. The used weak learner is a CART decision tree with maximum depth equal to 3 and decision

Table 1. Mean error rate obtained using 4 base classifiers

\begin{tabular}{|c|c|c|c|c|c|c|}
\hline Data Sets & SM & HM & SA & WA & BEST \\
\hline \multicolumn{7}{|c|}{ WRLC } \\
\hline Australian & $0.278(0.013)$ & $0.290(0.022)$ & $0.286(0.011)$ & $0.286(0.011)$ & $0.280(0.005)$ \\
\hline Balance & $0.197(0.023)$ & $0.206(0.034)$ & $0.309(0.032)$ & $0.330(0.025)$ & $0.410(0.015)$ \\
\hline Breast & $0.041(0.003)$ & $0.067(0.010)$ & $0.043(0.005)$ & $0.044(0.005)$ & $0.061(0.003)$ \\
\hline Cleveland & $0.244(0.010)$ & $0.265(0.014)$ & $0.249(0.011)$ & $0.254(0.009)$ & $0.279(0.011)$ \\
\hline Hayes & $0.325(0.017)$ & $0.361(0.023)$ & $0.353(0.019)$ & $0.354(0.016)$ & $0.371(0.016)$ \\
\hline Housing & $0.273(0.010)$ & $0.308(0.021)$ & $0.314(0.016)$ & $0.324(0.017)$ & $0.309(0.014)$ \\
\hline Ionosphere & $0.279(0.014)$ & $0.314(0.025)$ & $0.317(0.013)$ & $0.323(0.013)$ & $0.326(0.008)$ \\
\hline Liver & $0.432(0.010)$ & $0.436(0.013)$ & $0.446(0.011)$ & $0.449(0.009)$ & $0.437(0.005)$ \\
\hline Pima & $0.299(0.013)$ & $0.347(0.016)$ & $0.328(0.011)$ & $0.332(0.013)$ & $0.321(0.018)$ \\
\hline Sonar & $0.346(0.013)$ & $0.376(0.026)$ & $0.370(0.015)$ & $0.369(0.015)$ & $0.359(0.010)$ \\
\hline \multicolumn{7}{|c|}{ ADAR } \\
\hline Australian & $0.140(0.004)$ & $0.180(0.013)$ & $0.142(0.004)$ & $0.143(0.004)$ & $0.145(0.004)$ \\
\hline Balance & $0.080(0.004)$ & $0.096(0.007)$ & $0.078(0.004)$ & $0.078(0.004)$ & $0.089(0.004)$ \\
\hline Breast & $0.040(0.001)$ & $0.067(0.004)$ & $0.037(0.002)$ & $0.038(0.002)$ & $0.047(0.001)$ \\
\hline Cleveland & $0.202(0.009)$ & $0.247(0.015)$ & $0.227(0.009)$ & $0.226(0.009)$ & $0.240(0.006)$ \\
\hline Hayes & $0.215(0.011)$ & $0.278(0.026)$ & $0.211(0.010)$ & $0.209(0.010)$ & $0.240(0.016)$ \\
\hline Housing & $0.133(0.004)$ & $0.164(0.008)$ & $0.138(0.004)$ & $0.138(0.004)$ & $0.143(0.004)$ \\
\hline Ionosphere & $0.080(0.003)$ & $0.155(0.013)$ & $0.083(0.004)$ & $0.085(0.005)$ & $0.112(0.003)$ \\
\hline Liver & $0.285(0.009)$ & $0.334(0.020)$ & $0.286(0.011)$ & $0.289(0.011)$ & $0.321(0.010)$ \\
\hline Pima & $0.250(0.008)$ & $0.284(0.015)$ & $0.273(0.016)$ & $0.273(0.017)$ & $0.262(0.006)$ \\
\hline Sonar & $0.216(0.013)$ & $0.248(0.019)$ & $0.222(0.009)$ & $0.225(0.009)$ & $0.206(0.006)$ \\
\hline
\end{tabular}


Table 2. Mean error rate obtained using 7 base classifiers

\begin{tabular}{|c|c|c|c|c|c|c|}
\hline Data Sets & SM & HM & SA & WA & BEST \\
\hline \multicolumn{7}{|c|}{ WRLC } \\
\hline Australian & $0.272(0.009)$ & $0.298(0.017)$ & $0.287(0.009)$ & $0.288(0.009)$ & $0.286(0.009)$ \\
\hline Balance & $0.137(0.016)$ & $0.440(0.012)$ & $0.302(0.025)$ & $0.309(0.022)$ & $0.422(0.021)$ \\
\hline Breast & $0.040(0.002)$ & $0.084(0.010)$ & $0.040(0.003)$ & $0.041(0.004)$ & $0.062(0.001)$ \\
\hline Cleveland & $0.229(0.009)$ & $0.276(0.019)$ & $0.248(0.009)$ & $0.252(0.009)$ & $0.271(0.012)$ \\
\hline Hayes & $0.314(0.010)$ & $0.440(0.021)$ & $0.342(0.019)$ & $0.345(0.015)$ & $0.373(0.018)$ \\
\hline Housing & $0.268(0.008)$ & $0.303(0.031)$ & $0.323(0.014)$ & $0.333(0.016)$ & $0.294(0.017)$ \\
\hline Ionosphere & $0.261(0.011)$ & $0.320(0.033)$ & $0.315(0.009)$ & $0.316(0.008)$ & $0.321(0.007)$ \\
\hline Liver & $0.423(0.014)$ & $0.430(0.013)$ & $0.440(0.013)$ & $0.440(0.011)$ & $0.437(0.007)$ \\
\hline Pima & $0.291(0.080)$ & $0.358(0.018)$ & $0.324(0.008)$ & $0.325(0.008)$ & $0.313(0.017)$ \\
\hline Sonar & $0.348(0.010)$ & $0.361(0.017)$ & $0.382(0.009)$ & $0.387(0.009)$ & $0.357(0.009)$ \\
\hline \multicolumn{7}{|c|}{ ADAR } \\
\hline Australian & $0.137(0.003)$ & $0.214(0.013)$ & $0.145(0.002)$ & $0.143(0.003)$ & $0.145(0.004)$ \\
\hline Balance & $0.081(0.004)$ & $0.100(0.008)$ & $0.076(0.003)$ & $0.078(0.003)$ & $0.089(0.005)$ \\
\hline Breast & $0.040(0.001)$ & $0.073(0.006)$ & $0.036(0.001)$ & $0.036(0.001)$ & $0.046(0.001)$ \\
\hline Cleveland & $0.196(0.007)$ & $0.271(0.016)$ & $0.228(0.007)$ & $0.230(0.007)$ & $0.235(0.005)$ \\
\hline Hayes & $0.202(0.009)$ & $0.339(0.030)$ & $0.205(0.008)$ & $0.199(0.008)$ & $0.230(0.011)$ \\
\hline Housing & $0.130(0.006)$ & $0.184(0.011)$ & $0.137(0.003)$ & $0.137(0.003)$ & $0.143(0.004)$ \\
\hline Ionosphere & $0.079(0.003)$ & $0.174(0.011)$ & $0.080(0.003)$ & $0.081(0.005)$ & $0.114(0.004)$ \\
\hline Liver & $0.291(0.008)$ & $0.379(0.015)$ & $0.289(0.010)$ & $0.287(0.010)$ & $0.325(0.011)$ \\
\hline Pima & $0.247(0.004)$ & $0.287(0.010)$ & $0.280(0.014)$ & $0.280(0.013)$ & $0.267(0.006)$ \\
\hline Sonar & $0.215(0.005)$ & $0.253(0.016)$ & $0.221(0.007)$ & $0.222(0.009)$ & $0.207(0.005)$ \\
\hline
\end{tabular}

stumps as nodes functions. The number of boosting steps is equal to 10 . In order to obtain a lower correlation among different built classifiers, we have slightly modified the learning algorithm by providing an uniformly distributed random weight initialization. For this reason, we will refer to this classifier as ADAR (AdaBoost with Random weight inizialization). It is worth noting that in this way, we are considering two different scenarios for the classification models involved in the combination: a set of weak classifiers that independently would not achieve an high margin and a set of robust classifiers able to achieve high margins on the training set.

Our first experiment show the behavior of the margin-based fusion rule on the training set. To this aim, we considered the cumulative distributions of margins on the training set provided by SM and the other employed fusion rules. In fig. 1 we report the margin cdfs for the proposed approach in comparison with the other rules for the Housing data set when using 4 WRLC (fig. 1,a) or 4 ADAR (fig. 1,b) classifiers. Looking at these graphs, we can evaluate that the hard margin exhibits an higher minimum margin than our rule since we relax the constraint of the maximization problem to try to achieve better performance on the test set. It is also worth noting that the minimum margin evaluated by SM is always higher than SA and WA. 
In the second experiment, we have considered combinations with a number $K$ of dichotomizers equal to 4 and 7 . For each $K$, we have generated 30 different $K$-ples of dichotomizers and we have evaluated the mean error rate on the 10 runs of the hold out procedure for each combination. Tables 1, 2 we report the results obtained on the 10 data sets in terms of mean and standard deviation.

Let us analyze the results for WRLC classifiers (first half of the tables). In this case the soft margin rule is able to outperform the other rules in all the considered cases. When using ADAR classifiers (second half of the tables), instead, the results are slightly worse. There are two data sets (Breast and Hayes) where SA and WA are always better than SM independently on the number of combined classifiers. Moreover, when increasing to 7 classifiers even on Balance and Liver data sets WA and SA outperform SM. In all the other cases SM is better than the other rules (if we exclude Sonar data set where the BEST has higher performance than all the combined rules) and in particular, it is better than HM. This different behavior with ADAR is probably due to the better performance (both in terms of margin and generalization error) achieved by the base classifiers that lead to simple combination rules with high performance.

\section{Conclusions and Future Work}

We have proposed a framework for the linear combination of dichotomizers based on the margin maximization. Our approach relies on the evaluation of the weights of a convex combination of classifiers such that the margin of the combination on the training set is maximized. In the margin evaluation we relaxed the constraint using slack variables so as to achieve a bettergeneralization than the hard margin approach. The obtained results in comparisons with other recent and commonly employed approaches both on margin distribution and on classification performance encourage a deeper analysis of the proposed method. In particular, it could be useful to consider combination rules that maximize the average or the median margin rather than the minimum one. Moreover, a more extensive experimentation involving a real application (e.g. multibiometrics) could be employed to better verify the effectiveness of this approach.

\section{References}

1. Anthony, M., Bartlett, P.L.: Neural Network Learning: Theoretical Foundations. Cambridge University Press, Cambridge (1999)

2. Asuncion, A., Newman, D.J.: UCI machine learning repository (2007)

3. Bartlett, P.L.: The sample complexity of pattern classification with neural networks: the size of the weights is more important than the size of the network. IEEE Transactions on Information Theory 44(2), 525-536 (1998)

4. Breiman, L.: Prediction games and arcing algorithms. Neural Computation 11(7), 1493-1517 (1999)

5. Crammer, K., Gilad-Bachrach, R., Navot, A., Tishby, N.: Margin analysis of the LVQ algorithm. In: Advances in NIPS, vol. 15, pp. 462-469 (2003) 
6. Fumera, G., Roli, F.: A theoretical and experimental analysis of linear combiners for multiple classifier systems. IEEE Transactions on Pattern Analysis and Machine Intelligence 27(6), 942-956 (2005)

7. Grove, A.J., Schuurmans, D.: Boosting in the limit: maximizing the margin of learned ensembles. In: Proc. AAAI 1998/IAAI 1998, pp. 692-699. American Association for Artificial Intelligence (1998)

8. Jain, A.K., Nandakumar, K., Ross, A.: Score normalization in multimodal biometric systems. Pattern Recognition 38(12), 2270-2285 (2005)

9. Koltchinskii, V., Panchenko, D.: Empirical margin distributions and bounding the generalization error of combined classifiers. Annals of Statistics 30, 1-50 (2002)

10. Kuncheva, L.I.: Combining Pattern Classifiers. Methods and Algorithms. John Wiley \& Sons, Chichester (2004)

11. Kuncheva, L.I., Whitaker, C.J.: Measures of diversity in classifier ensembles. Machine Learning 51, 181-207 (2003)

12. Marrocco, C., Ricamato, M.T., Tortorella, F.: Exploring margin maximization for biometric score fusion. In: da Vitoria Lobo, N., Kasparis, T., Roli, F., Kwok, J.T., Georgiopoulos, M., Anagnostopoulos, G.C., Loog, M. (eds.) S+SSPR 2008. LNCS, vol. 5342, pp. 674-683. Springer, Heidelberg (2008)

13. Ratsch, G., Onoda, T., Muller, K.R.: Soft margins for adaboost. Machine Learning 42(3), 287-320 (2001); NeuroCOLT Technical Report NC-TR-1998-021

14. Schapire, R.E., Freund, Y., Bartlett, P., Lee, W.S.: Boosting the margin: A new explanation for the effectiveness of voting methods. The Annals of Statistics 26(5), 1651-1686 (1998)

15. Shawe-Taylor, J., Bartlett, P.L., Williamson, R.C., Anthony, M.: Structural risk minimization over data-dependent hierarchies. IEEE Transactions on Information Theory 44(5), 1926-1940 (1998)

16. Tumer, K., Ghosh, J.: Analysis of decision boundaries in linearly combined neural classifiers. Pattern Recognition 29, 341-348 (1996)

17. Vanderbei, R.J.: Linear Programming: Foundations and Extensions, 2nd edn. Springer, Heidelberg (2001)

18. Vapnik, V.N.: Statistical Learning Theory. John Wiley \& Sons, Chichester (1998)

19. Vezhnevets, A., Vezhnevets, V.: Modest adaboost - teaching AdaBoost to generalize better. In: Graphicon-2005, Novosibirsk Akademgorodok, Russia (2005) 